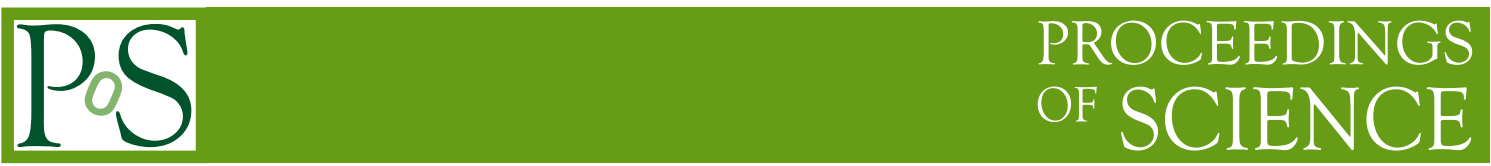

\title{
Cosmological results from the Kilo Degree Survey
}

\author{
Hendrik Hildebrandt* \\ Argelander-Institut für Astronomie, Universität Bonn, Auf dem Hügel 71, 53121 Bonn, Germany \\ E-mail: hendrikeastro.uni-bonn.de
}

on behalf of the KiDS collaboration

\begin{abstract}
Gravitational lensing represents a unique tool to study the dark Universe. In the weak lensing regime small distortions in the images of galaxies caused by the large-scale structure can be detected over the whole sky. Measuring these coherent distortions yields cosmological insights complementary to other probes like the cosmic microwave background (CMB). Ongoing wide-field imaging surveys exploit this to come up with competitive constraints on important cosmological parameters. Here we concentrate on recent results from the ongoing European Kilo Degree Survey (KiDS) that show a mild tension with $\mathrm{CMB}$ measurements from the Planck mission when the standard cosmological model is assumed. Possible solutions to this discrepancy using extensions to the standard model of cosmology and future developments are discussed.
\end{abstract}

The European Physical Society Conference on High Energy Physics

5-12 July, 2017

Venice

${ }^{*}$ Speaker. 


\section{Introduction}

The standard model of cosmology, characterised by a cosmological constant $\Lambda$ and cold dark matter (hence its name $\Lambda \mathrm{CDM}$ ), has been established almost two decades ago and has so far succeeded in describing a large variety of cosmological measurements. Observations have progressed tremendously and challenge this concordance model with rapidly increasing precisionmeasurements. The weak lensing effect of the large-scale-structure, called cosmic shear, has evolved into a major cosmological probe. This effect can be measured from the minute distortions (shear) of large numbers of galaxy images observed in wide-area imaging surveys of the sky. Correlating the ellipticities of pairs of galaxies and modelling the signal according to $\Lambda$ CDM can yield competitive and complementary constraints on some of the most important cosmological parameters like the total matter density, $\Omega_{\mathrm{m}}$, the amplitude of the matter power spectrum, $\sigma_{8}$, and most precisely - their combination, parametrised as $S_{8}=\sigma_{8} \sqrt{\Omega_{\mathrm{m}} / 0.3}$.

The most important observational challenges are to measure ellipticities and redshifts of millions of galaxies with very small biases. The redshifts for cosmic shear observations come from photometric observations and are dubbed photo- $z$. These photo- $z$ have to be calibrated extremely well, which usually involves deep spectroscopic calibration samples.

Comparing the results from cosmic shear measurements over the past decade to the best measurements from the cosmic microwave background [1] reveals a slight discrepancy in the values of the $S_{8}$ parameter [2]. Weak lensing measurements have yielded $S_{8}$ values that are consistently lower than the arguably very robust Planck value of $S_{8}=0.851 \pm 0.024$. One possibility for this discrepancy is that there are systematic errors in the cosmic shear measurements that haven't been taken fully into account yet. With new measurements from the Kilo Degree Survey (KiDS), which are presented here, we made significant progress in this area and were able to decrease some of the most important systematic uncertainties to make cosmic shear measurements more robust and comparisons with the $\mathrm{CMB}$ more reliable.

\section{The Kilo Degree Survey (KiDS)}

Using the OmegaCam instrument mounted at the Cassegrain focus of ESO's VLT Survey Telescope (VST) on Cerro Paranal in Chile the KiDS collaboration aims at observing 1350 $\mathrm{deg}^{2}$ of the sky in four optical bands ugri. Compared to other past and ongoing imaging surveys KiDS has three main advantages:

1. The VST is the first professional telescope that was designed with the weak lensing science case in mind. Hence it was optimised to show benign optical distortions because these distortions have to be corrected for before ellipticities can be measured accurately.

2. The depth of KiDS is well-matched with the deepest spectroscopic surveys. Hence the KiDS photo- $z$ can be directly calibrated with spectroscopic redshifts (spec- $z$ ) which isn't possible for deeper surveys.

3. KiDS is complemented by an infrared survey called VIKING (VISTA Kilo Degree Infrared Galaxy Survey) that adds images in five more near-infrared (NIR) passbands, $Z Y J H K_{\mathrm{s}}$. This unique combination of an optical and NIR survey will eventually yield the best possible photo- $z$, which are required for the most robust cosmological measurements with cosmic shear. 
Here we present results from a third of the KiDS data that was available in 2015 (called KiDS450 due to its area of $\sim 450 \mathrm{deg}^{2}$ ). We also give an outlook on how we will improve the cosmic shear analysis when adding the VIKING data as well as more area over the next years.

\section{Tomographic cosmic shear measurements}

In [3] we used the KiDS optical 4-band photometry to estimate photo- $z$ and divide 12.5 million galaxies into four tomographic photo- $z$ bins in the range $0.1<z_{\text {phot }} \leq 0.9$. The actual redshift distributions that are needed for an accurate modelling of the cosmic shear signal were estimated with three different, redundant approaches, based on a spec- $z$ calibration sample.

Ellipticities of all these galaxies were measured from $r$-band images with a Bayesian forwardfitting model technique that self-calibrates the measurements at runtime. Any residual biases in the shape measurements are estimated and corrected for via a large suite of image simulations that mimic many of the observational aspects of the survey data.

The uncertainties on the redshift and shear calibration are fully propagated into the cosmological results and marginalised over, e.g. when values of $S_{8}$ are presented below. Furthermore, we introduced a blinding scheme to suppress confirmation bias in the analysis. For this we sent the catalogue of galaxy ellipticities to an external "blind-setter" who coherently perturbed the ellipticities. Two perturbed catalogues were sent back to us along with the unperturbed catalogue without revealing their identities. The cosmological analysis was carried out with all three catalogues and unblinding occurred only at the very end, shortly before submission of the paper to the journal. Most importantly, this unblinding happened after the analysis pipeline had been frozen.

\section{Results}

In Fig. 1 the main cosmological result from the KiDS-450 tomographic cosmic shear measurement is presented, showing constraints on $\Omega_{\mathrm{m}}$ and $\sigma_{8}$ as well as their combination $S_{8}$ (which is essentially uncorrelated to $\Omega_{\mathrm{m}}$ ). Also shown are results from CFHTLenS (Canada France Hawaii Telescope Lensing Survey [4]), a combined pre-Planck CMB analysis [5], and [1].
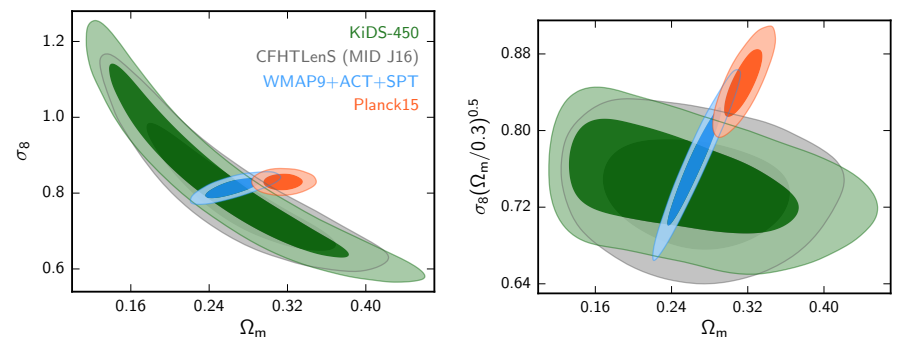

Figure 1: Constraints on $\Omega_{\mathrm{m}}$ and $\sigma_{8}$ (left) and $\Omega_{\mathrm{m}}$ and $S_{8}$ (right) from KiDS450 [3], CFHTLenS (Canada France Hawaii Telescope Lensing Survey [4]), a combined pre-Planck CMB analysis [5], and [1]. (figure taken from [3])

The general picture has not changed. KiDS-450, although in terms of systematic errors arguably much more robust than previous cosmic shear measurements, still yields a lower value of $S_{8}=0.745 \pm 0.039$ than what is measured by Planck. The value is in very good agreement with pre-Planck CMB measurements as well as cosmic shear constraints from CFHTLenS though. 
The discrepancy between the Planck and KiDS measurements is at the $2.3 \sigma$ level if the full highdimensional parameter space is considered. While this does not constitute a strong disagreement of these two cosmological probes it certainly deserves attention and needs to be understood.

\section{Extended cosmologies}

The most probable reason for the discrepancy between Planck and KiDS is a systematic error in one of the two probes (or both). The significance is low so that no extraordinary claims about deviations from $\Lambda \mathrm{CDM}$ are really justified at this point. However, it is still interesting to test whether the discrepancy holds for plausible extensions to the base $\Lambda$ CDM model. In [6] we have tested several such extensions, including non-flat cosmologies, universes with massive neutrinos, static and evolving (in terms of the equation-of-state) dark energy models, modified gravity scenarios, and a running of the spectral index of the primordial power spectrum. The only extended model that alleviates the tension between Planck and KiDS and at the same time is mildly preferred by the combined KiDS+Planck data set is an evolving dark energy model. Constraints on $\Omega_{\mathrm{m}}$ and $\sigma_{8}$ as well as the dark energy equation-of-state parameters $w_{0}$ and $w_{a}$ are shown in Fig. 2.
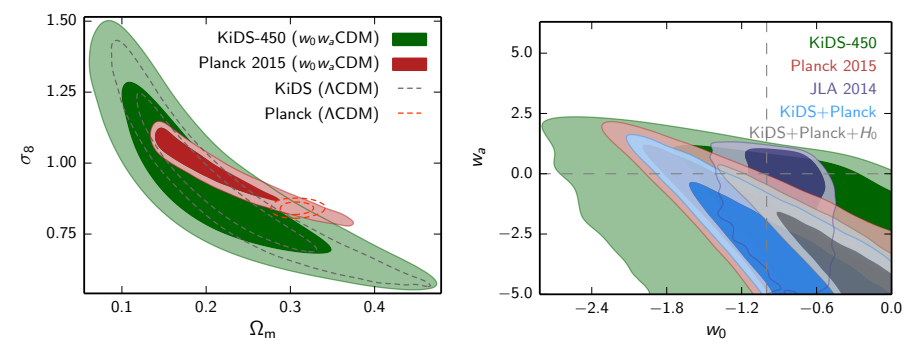

Figure 2: Constraints on $\Omega_{\mathrm{m}}$ and $\sigma_{8}$ (left) and the dark energy equation-ofstate parameters $w_{0}$ and $w_{a}$ (right) from KiDS and Planck in an evolving dark energy model. (figure taken from [6])

Combining KiDS cosmic shear measurements with measurements from galaxy redshift surveys yields very interesting results as well. When clustering and galaxy-galaxy lensing (GGL) results from the overlapping GAMA survey are combined with the KiDS cosmic shear measurements [7] the cosmological constraints do not only become more precise but also show a slightly larger value of $S_{8}$, more compatible with the Planck value. In contrast to this result, combining KiDS cosmic shear with clustering and GGL measurements from BOSS and 2dFLenS yields a low $S_{8}$ [8]. Similarly, a low $S_{8}$ is found by the Fourier-space cosmic shear analysis of the KiDS- 450 data by [9]. At the moment these differences aren't fully understood and require more research.

\section{Summary and outlook}

In [3] we used data from KiDS to measure tomographic cosmic shear and constrain cosmological parameters. Emphasising the work on the most prominent systematic effects we came up with one of the arguably most robust cosmic shear measurements to date. Similar to many previous cosmic shear results our $S_{8}$ measurement yields a lower value than the most recent measurement based on Planck CMB data. This discrepancy is currently not understood and requires further research into systematic errors as well as possible extensions to the standard model of cosmology.

A major improvement in future KiDS measurements will be the addition of NIR data from the VIKING project. Combining KiDS and VIKING will not only yield more precise photo- $z$ (see 
Fig. 3) but it will also allow us to measure cosmic shear at higher redshifts, where the cosmic shear signal is particularly strong. This larger redshift baseline as well as the growing areal coverage will further decrease the statistical uncertainties. But maybe most importantly the optical+NIR data will allow for a much more robust redshift calibration, hence reducing one of the most important systematic errors as well. It remains to be seen whether those measurements over the next years reveal some systematic cause for the mild discrepancies that we are seeing now or solidify the tension and potentially signify the breakdown of the standard model of cosmology.
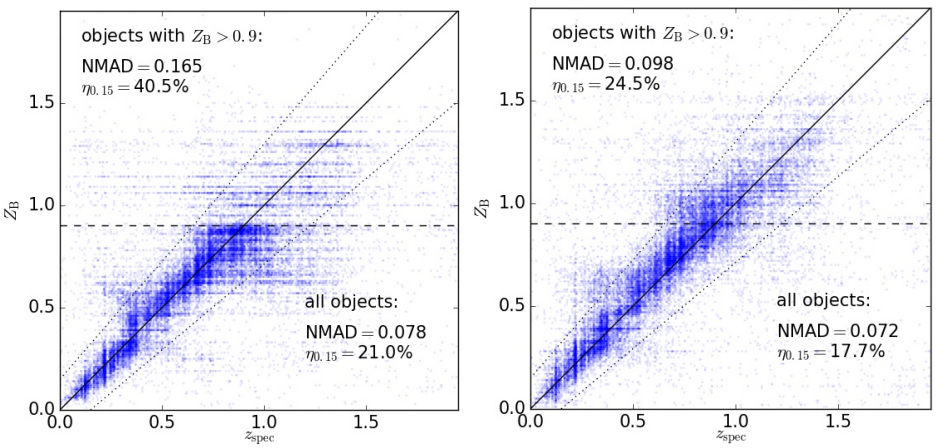

Figure 3: Photometric redshifts $\left(Z_{\mathrm{B}}\right)$ vs. spectroscopic redshifts $\left(z_{\text {spec }}\right)$ for KiDS 4-band optical data (left) and KiDS+VIKING 9-band optical+NIR data (right). NMAD is the normalised median absolute deviation and $\eta_{0.15}$ is the fraction of outlier objects with $\left|Z_{\mathrm{B}}-z_{\text {spec }}\right| /\left(1+z_{\text {spec }}\right)>0.15$. (figure taken from Hildebrandt et al. in prep.)

\section{References}

[1] Planck Collaboration, P. A. R. Ade, N. Aghanim, M. Arnaud, M. Ashdown, J. Aumont et al., Planck 2015 results. XIII. Cosmological parameters, A\&A 594 (Sept., 2016) A13, [1502. 01589].

[2] M. Kilbinger, Cosmology with cosmic shear observations: a review, Reports on Progress in Physics $\mathbf{7 8}$ (July, 2015) 086901, [1411.0115].

[3] H. Hildebrandt, M. Viola, C. Heymans, S. Joudaki, K. Kuijken, C. Blake et al., KiDS-450: cosmological parameter constraints from tomographic weak gravitational lensing, MNRAS 465 (Feb., 2017) 1454-1498, [1606.05338].

[4] C. Heymans, E. Grocutt, A. Heavens, M. Kilbinger, T. D. Kitching, F. Simpson et al., CFHTLenS tomographic weak lensing cosmological parameter constraints: Mitigating the impact of intrinsic galaxy alignments, MNRAS 432 (July, 2013) 2433-2453, [1303.1808].

[5] E. Calabrese, R. A. Hlozek, N. Battaglia, E. S. Battistelli, J. R. Bond, J. Chluba et al., Cosmological parameters from pre-planck cosmic microwave background measurements, Phys. Rev. D 87 (May, 2013) 103012, [1302.1841].

[6] S. Joudaki, A. Mead, C. Blake, A. Choi, J. de Jong, T. Erben et al., KiDS-450: Testing extensions to the standard cosmological model, arXiv:1610.04606 (Oct., 2016) .

[7] E. van Uitert, B. Joachimi, S. Joudaki, C. Heymans, F. Köhlinger, M. Asgari et al., KiDS+GAMA: Cosmology constraints from a joint analysis of cosmic shear, galaxy-galaxy lensing and angular clustering, arXiv:1706.05004 (June, 2017) .

[8] S. Joudaki, C. Blake, A. Johnson, A. Amon, M. Asgari, A. Choi et al., KiDS-450 + 2dFLenS: Cosmological parameter constraints from weak gravitational lensing tomography and overlapping redshift-space galaxy clustering, arXiv:1707.06627 (July, 2017) .

[9] F. Köhlinger, M. Viola, B. Joachimi, H. Hoekstra, E. van Uitert, H. Hildebrandt et al., KiDS-450: the tomographic weak lensing power spectrum and constraints on cosmological parameters, MNRAS 471 (Nov., 2017) 4412-4435, [1706.02892]. 